\title{
Key Priority Research Areas towards Disaster Risk Reduction
}

\author{
Sakiko KANBARA \\ University of Kochi, Graduate School of Nursing, Kochi, Japan
}

\begin{abstract}
This paper delineates the key issues as highlighted at a speech made at the Health Emergency and Disaster Nursing (HEDN) Seminar on February 11th, 2021. The purpose of the discussions is to share disaster insights and identify key priority research areas towards Disaster Risk Reduction to know disaster nursing, and the research needed for the future global agenda, and which nursing may have already contributed, that way forward for understanding risk focused on capacity and vulnerability.
\end{abstract}

\section{INTRODUCTION}

This paper delineates the key issues as highlighted at a speech made at a seminar convened by the Editorial Office of the Health Emergency of Disaster Nursing on February 11th, 2021. The purpose of the discussions is to monitor disaster nursing for young academia, to share disaster insights and identify key priority research areas towards Disaster Risk Reduction (DRR) in the region. The way forward for understanding risk focused on capacity and vulnerability; these were priority areas identified and are discussed in this paper. The first part is dedicated to history, the trends of research in the field of disaster nursing, and the research needed for the future global agenda, disaster countermeasures, and disaster risk-reduction measures implemented, to which nursing may have already contributed.

\section{TRAJECTORY OF DISASTER NURSING RESEARCH}

The story of Nightingale, which marked the beginning of modern nursing, is a famous one, but Nightingale not only held a lamp and came to the aid of people, but also

Correspondence: Sakiko Kanbara, University of Kochi, Graduate School of Nursing, 2751-1, Ike, Kochi, 781-8515 Japan. Email: kanbara@cc.u-kochi.ac.jp

Received 4 March 2021; accepted 5 March 2021; J-STAGE advance published 6 April 2021.

This article provides highlights from a lecture that was given at the Health Emergency and Disaster Nursing (HEDN) Seminar held on February 11, 2021. This seminar was supported by the SECOM Science and Technology Foundation. demonstrated the importance of infographics and statistics. Before 1995, there was no research about "disaster nursing". Regarding the history of disaster nursing research, in Japan, the Great Hanshin-Awaji Earthquake triggered the establishment of the Japan Society of Disaster Nursing. Various disaster nursing research was conducted using the lessons learned from the earthquake. Nursing is a widely complex profession and is used diversely during disaster situations. In disaster medicine, evidence for Disaster Medical Assistance Teams (DMAT) establishment came when Aoki et al. insisted that if the emergency rescue had been conducted earlier and the right people had been brought to the right hospitals, so many lives could have been saved; this is called preventable death. However, it was too difficult to obtain original disaster samples or data that were acceptable for analyses in 2004.

In 2007, the University of Hyogo was conducting research on the construction of disaster nursing in the ubiquitous society. Research funding was an issue that needed to be addressed. As result of the Delphi study in 2007, it was revealed that mental health and hospital preparedness were the most popular topics to be studied. However, the Delphi study report was published in 2010; therefore, it was difficult to say how much research was completed to be effective in the March 11 disaster, the Great East Japan Earthquake. In fact, there was a need for a Delphi study on special disasters, especially nuclear disasters, but further research on international disasters and complex disasters had not been conducted yet. In the aftermath of the Great East Japan Earthquake, it was necessary to take a long-term perspective on reconstruction and social participation, especially in terms of a 


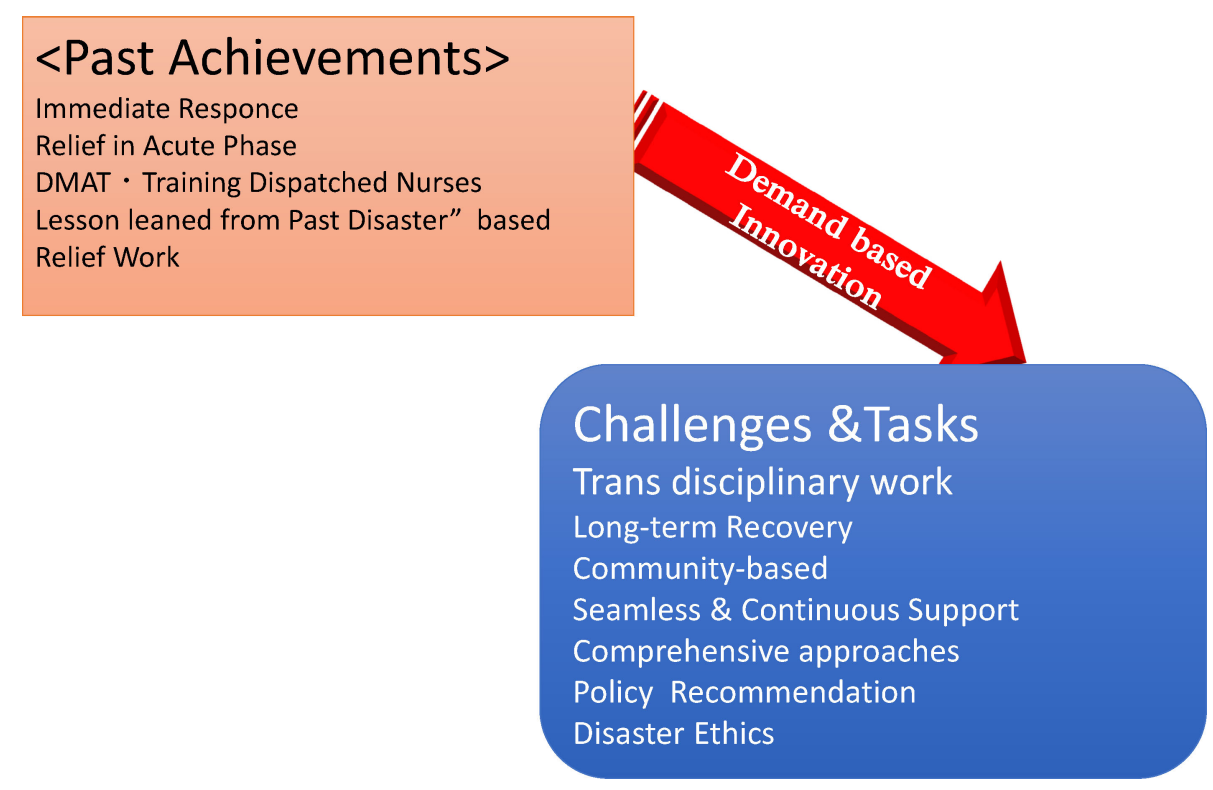

Figure 1 Demand-based innovation of disaster nursing after 2011.

wide-area disaster. Since 2013, there has been an increase in the number of research funds available. Whenever a disaster occurs, research increases. It started with basic nursing and spread to community nursing and gerontological nursing with education, preparation, and community activities involved thereafter. Research areas have been expanded to disaster mitigation, disaster prevention, and resilience, but there is a possibility that the people in the field of resilience and disaster prevention are not disaster nurses or nursing researchers. There are no promising research project Grants-in-Aid for Scientific Research (A) funded by the Japan Society for the Promotion of Science (JSPS), which budget over 200,000 USD per year; however, the number of basic research projects conducted by early career researchers, $<8$ years after completing their $\mathrm{PhD}$, is increasing and more grants are available to them.

\section{CAPACITY BUILDING FOR DISASTER NURSING RESEARCH}

Japan is exposed to a wide variety of disasters, and the nursing profession is in a position to conduct research under such circumstances, which means that Japan is in a position to take a worldwide leadership role. The number of both of nursing professionals and nursing colleges has been increased rapidly. In addition, the number of fulltime disaster nursing faculty members has been increasing rapidly over the past 10 years. To establish the Disaster
Nursing Global Leadership Program (DNGL) 2012, there were many discussions about what types of leaders are needed in the future. Since 2011, disasters have become more complex, diversified, and prolonged, even in other countries, and there has also been a shift from an acute to medium- to long-term disaster response, a single-hazard disaster response, and a shift to community-based disaster nursing in which the responsibility is shared more broadly with the community (Figure 1). In the aftermath of the Great East Japan Earthquake, in Kochi, Wakayama, and Kagoshima, countermeasures against the Nankai Trough and an earthquake directly beneath the Tokyo metropolitan area have been required, and in the midst of these countermeasures against large-scale disasters, a major flood has occurred, and it occurs every year. Emergency life-saving measures are of course necessary when people's lives are threatened.

\section{JOURNAL OF THE JAPAN SOCIETY OF DISASTER NURSING AND THE 2020 ANNUAL CONFERENCE}

Figure 2 shows the key words of the Journal of the Japan Society of Disaster Nursing. On the left are the key words used in original papers, and on the right are the key words from the conference presentations that were given at the annual conference in 2020. At the conference, there was the opportunity to discuss matters related to coronavirus (COVID-19); it was a good opportunity to discuss such a 


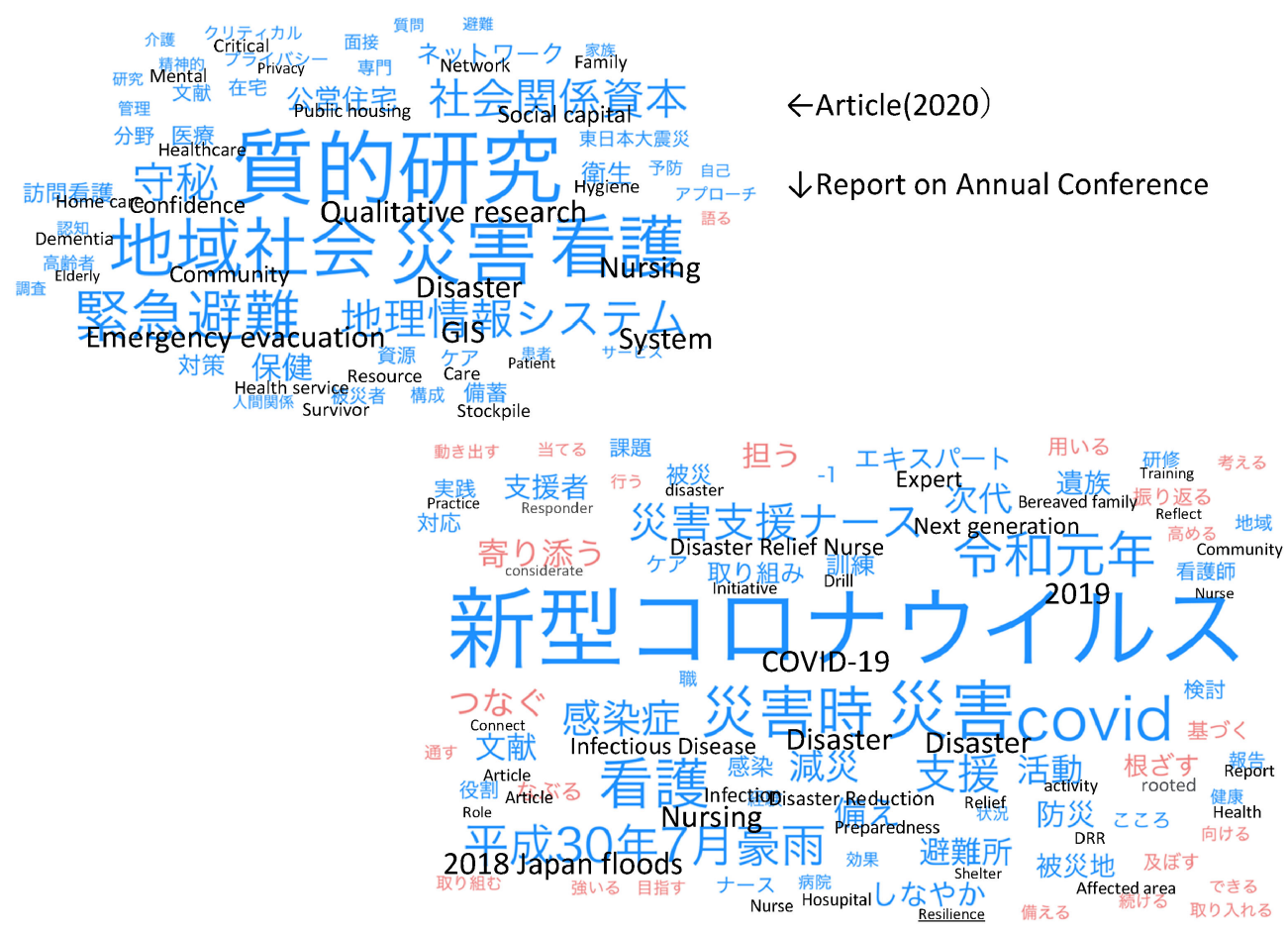

Figure 2 Text mining analysis from key words of original articles (left) and from the annual disaster nursing conference (right).

timely topic. It will be interesting to see how many papers will be published on this topic and it will be a challenge in the future to determine what can be done to publish papers at the same speed as it takes to report on updates on this interesting topic. Various topics such as geographic information system, emergency evacuation, society, and social capital can be researched, all of which require time. The gap between time to practice, research and publish must be minimized in order to update the community in a timely manner.

\section{IMPROVEMENTS IN RESEARCH AND INTERDISCIPLINARY COLLABORATION}

One of the barriers in disaster nursing is the lack of pilot studies or pre-disaster studies. The sources of data are not always the same. When we try to publish this information in general clinical journals, we are often asked if the readers will be the right "audience" or if the methodology is too complicated. In addition, there are situations where research cannot be conducted unless there are equal co-researchers in the field; co-researchers who can be trusted by both providers and receivers of support. There is also a lack of budget. As mentioned earlier, it is difficult to find the funding to quickly come up with a disaster research plan when it is suddenly necessary. It is necessary to work together with other research areas such as engineering. In this context, ethical considerations, and effectiveness and reliability must be constantly considered as society changes. Incidentally, in terms of COVID-19, International Council of Nurses (ICN's) nursing guidelines had not been revised since 2013 because of the need to improve and revise the Code of Ethics amidst the use of various technologies, various social changes, and the so-called new normal, as well as the conflicts before that. I have heard that the Code of Ethics is currently being debated, in terms of how to respond to the changes in technology that can adapt to the new society and the changing sense of ethics in various societies. In Japan, the Science Council of Japan has established the Academic Collaboration Team for Disaster Reduction, and 54 academic societies are conducting research on various aspects of disaster response. In such a situation, the role of nursing is to conduct research in nursing research, the essence of nursing, and then publish that research into journals, so that it can be useful for research in other fields, and so we can conduct research while using research from other fields. I believe that transdisciplinary research will be 


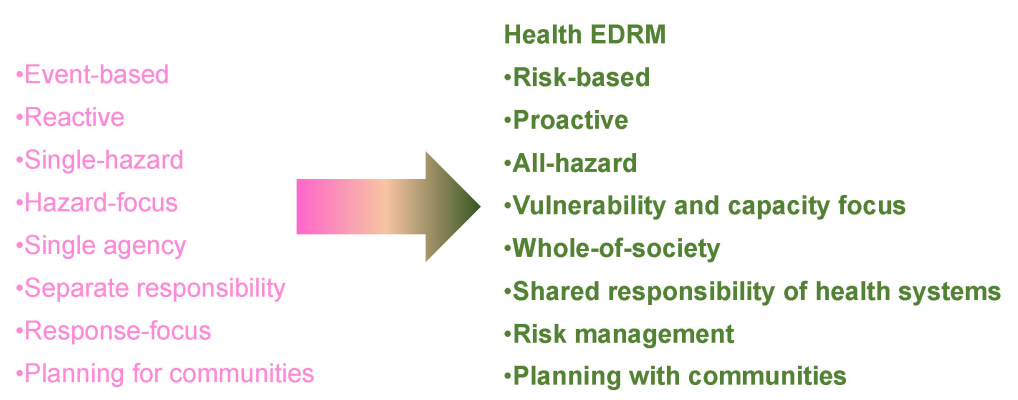

Figure 3 Health emergency and disaster risk management framework.

required in the future. What I think is difficult for human science in this context is that people are mobile, and infrastructure is uncertain. In this way, data itself is difficult to access, including that of communication between local nurses and people in the field. Even so, nurses are the ones who can approach the data that are closest to the core. However, I think we need to think about how to access that data. The Sustainable Development Goals (SDGs) say that we should not overlook vulnerable people, so I think we need to think about how much we can do to ensure that no one is left out.

\section{WAY FORWARD}

Figure 3 is a framework for health emergencies and disaster risk management issued by the World Health Organization (WHO, 2019). It does not consider single hazards within a single framework, but rather, it considers what type of risks are associated with the disaster, such as the Great East Japan Earthquake. We should think about the risk of water shortage, job loss, injury, and so on, and we should think about it on a riskby-risk basis, and we should take a more proactive approach to prevention and risk reduction, rather than retroactively responding to a disaster event; we should think about all hazards, not just one hazard at a time. Also, instead of focusing on hazards, as mentioned earlier in the disaster risk equation, we should focus more on vulnerability and capacity, and think of this as a problem for society as a whole, not a problem for a single health center or the Cabinet Office. This means that the responsibility should be shared, not by one party, but by the entire system. This is a matter of adjusting one's thinking, though.

Instead of focusing on the disaster response, we need to focus on risk management and have a managementbased approach. And instead of "planning for the community", we should plan with the community. This framework is designed to be more interdisciplinary than the WHO's health emergencies and disaster risk management strategy, and it is designed to work with the people. For disaster nursing, it is important to bring together the research that has already been conducted and make it clear what needs to be done in the future for this framework.

The Sendai Framework for Disaster Reduction emphasizes the importance of all-hazards, people-centered, and people-oriented approaches. Disaster nursing should refer to the Sendai Framework for Disaster Reduction and determine whether health emergencies are being properly researched and whether the measures are having an impact on practice, whether they are being properly included in disaster prevention policy plans, and whether they are being developed into guidelines that take into consideration the younger generation. In order to incorporate this into the research, it is not enough to say that the recommendations or the inclusion of the framework are meaningful or necessary; they are both required. It does not have to be something that can be turned into a perfect policy or guideline, but it is important to think about disaster nursing that can be controlled in various places at the conceptual level.

In disaster risk management, there is still a lack of knowledge about health, so it is important to improve health from the outside, especially in the case of COVID19. In particular, during COVID-19, disaster nurses were called upon to work in various places, and I think this is a field that can bridge the gap between knowledge and practice. In addition, we have global partnerships, and this is one of the strengths of disaster nursing research that we need to build on. This gap needs to be addressed and research should be enhanced, so we are able to discuss the awareness of this issue through research publications or through webinars. There are many of aspects about disaster nursing that were not thought of before the COVID-19 pandemic. 


\section{DISCLOSURE}

The terms of this article have been reviewed and approved in accordance with policy on objectivity from the Japan Society of Disaster Nursing.

\section{REFERENCE}

World Health Organization (2019). Health Emergency Disaster Risk Management Framework [Cited 27 Feb 2021.] Available from URL: https://www.who.int/hac/techguidance/preparedness/ health-emergency-and-disaster-risk-management-frameworkeng.pdf 